\title{
The Efficacy, Safety and Tolerability of Retapamulin as a Treatment Option for Impetigo and Other Uncomplicated Superficial Skin Infections: A Meta-analysis
}

\author{
Rudy Ciulianto ${ }^{1, *}$ \\ ${ }^{1}$ Departement of Pediatrics, Faculty of Medicine, Krida Wacana Christian University, Jl. Terusan Arjuna Utara No.6 Jakarta 11510, Indonesia \\ *Corresponding author. E-mail: rudy.chow@ukrida.ac.id
}

\section{Abstract}

$\mathrm{B}$ ACKGROUND: The treatment of impetigo, secondarily infected dermatitis, and infected traumatic lesions continue to develop as new generations of drugs are being formulated. Bacteria causing impetigo show growing resistance rates for commonly used antibiotics. Retapamulin being a new drug has been recently approved as topical antibiotic in children and adult. This study aimed to ascertain the efficacy, safety and tolerability of retapamulin as the treatment option for impetigo and other uncomplicated superficial skin infections.

METHODS: A search for studies published from 2006-2014 was done in Pubmed, EBSCO, OVID, Science Direct, and Cochrane using the search strategy. The search was limited to studies conducted in human subjects and published in the English language. Randomized controlled trials evaluating the efficacy, safety and tolerability of retapamulin as treatment for impetigo and other uncomplicated superficial skin infections in children and adult were included and extracted independently and the qualities of the studies were appraised using critical appraisal tools. Data analysis was conducted by using RevMan 5 .

RESULTS: This study has high heterogeneity and found Retapamulin has no statistically significant difference in the clinical success after seven days and follow up among per-protocol-patients, bacteriogical confirmed patients and intention-to-treat patients with impetigo and other secondary infected traumatic lesions compared to other regimens. However, Retapamulin has beneficial effect in the clinical success, well tolerated and safe for children and adults.

CONCLUSION: Retapamulin is comparably effective and safe as a treatment option for impetigo and other uncomplicated superficial skin infections.

KEYWORDS: efficacy, safety, tolerability, retapamulin, impetigo, meta-analysis

Indones Biomed J. 2015; 7(1): 49-56

\section{Introduction}

Primary skin infections, such as impetigo, develop as a direct result of pathogen invasion of otherwise healthy skin.(1) Impetigo is one of the most common and higly contagious examples of primary skin infections.(2) Staphylococcus aureus is the predominant infection organism in impetigo (isolated in $70 \%$ of cases), either as a sole infecting organism (bullous impetigo) or in combination with Streptococcus pyogenes (non-bullous impetigo).(2-4) Infection with S. pyogenes alone is less common.(2) Impetigo is most frequently seen in children aged $2-5$ years; $(2,4,5)$ however, individuals of all ages, races and genders are susceptible to impetigo.(5)

Secondary skin infections develop following trauma to the skin.(1) Examples include secondarily infected traumatic lesions, which may follow abrasions, lacerations or suture 
wounds, and secondarily infected dermatitis (eczema). Secondarily infected traumatic lesions may subsequently develop abscesses.(3) S. aureus is the most common pathogen associated with the majority of uncomplicated secondary superficial skin infections, followed by $\mathrm{S}$. Pyogenes.(4)

Although uncomplicated superficial infections do not usually progress to serious infections, they do warrant concern, especially in paediatric populations in whom these infections occur frequently (6) and in the elderly who are more susceptible than younger individuals.(7)

The use of topical antibacterials is recommended in the treatment of uncomplicated superficial skin infectious, where the lesions are localized and limited in numbers. (2,4,5,7-9) Until recently, the only topical antibacterial agents widely recommended for skin infections were fusidic acid (10) and mupirocin $(11,12)$. Emerging resistance to topical fusidic acid (13-15) and to mupirocin (16-18) has prompted the development of new antibacterials.

Topical retapamulin 1\% ointment (Altargo ${ }^{\circledR}$ ) (1) is the first antibacterial of the pleuromutilin class approved for use in humans; $(19,20)$ other pleuromutilins, such as tiamulin,(21) are employed for veterinary use. Retapamulin is approved for the treatment of impetigo $(19,20)$, infected small lacerations, abrasions or sutured wounds without abscesses. (19)

Impetigo is highly contagious and easily spreads from person to person by touch. Impetigo is thought to be most common between the ages of two and six. About one in 35 children under the age of four and one in 60 children under 15 develop impetigo. In a survey of Dutch children it was the third most frequent skin condition, after eczema and viral warts. It's thought to be more common in tropical and developing countries.

Over recent decades physician visits for cellulitis and soft-tissue infections have increased from 32 to 48 per 1.000 population from 1997 to 2005 . Necrotising fasciitis caused by group A streptococci is now endemic in the USA. Of great concern is that Staphylococcus aureus, the predominant cause of cellulitis, and abscesses or wound exudates, are becoming increasingly resistant to methicillin. Vancomycin and other newer antibiotics are therefore the drugs of choice. (5) This is true even for community-acquired methicillinresistant $\mathrm{S}$. aureus (MRSA), demonstrating that MRSA is no longer a pathogen limited to hospitalised patients.(22)

The general objective of this study is to ascertain the efficacy, safety and tolerability of retapamulin as the treatment option for impetigo and other uncomplicated superficial skin infections. In this study also able to verify the clinical response and the adverse effects of retapamulin in management of impetigo and other uncomplicated superficial skin infections compare to other drugs.

\section{Methods}

\section{Criteria for Considering Studies for This Review Types of Studies}

Included in this review were randomized control trials on the use of retapamulin in the treatment of impetigo and other uncomplicated superficial skin infections.

\section{Types of Participants}

Included children and adult who have impetigo, secondarily infected dermatitis, and secondarily infected traumatic lesions of the skin.

\section{Types of Interventions}

The intervention in the included studies is use of:

1) Topical retapamulin ointment twice daily for 5 days versus oral cephalexin twice daily for 10 days in the treatment of secondarily infected dermatitis.

2) Topical retapamulin ointment twice daily for 5 days versus oral cephalexin twice daily for 10 days for empiric treatment of secondarily infected traumatic lesions of the skin.

3) Topical retapamulin ointment twice daily for 5 days versus sodium fusidate ointment 3 times daily for 7 days for impetigo.

4) Topical retapamulin ointment twice daily for 5 days versus topical placebo as treatment of impetigo.

We excluded studies that only compared different dosages of the same drug.

\section{Search Methods for Identification of Studies}

A search for studies published from 2006-2014 was done in Pubmed, EBSCO, OVID, Science Direct, and Cochrane using the search strategy. The search was limited to studies conducted in human subjects and published in the English language.

\section{Data Collection and Analysis}

Data were extracted independently by two reviewers. Critical appraisal of the methodology and the outcome measures were also done. Whenever there are disagreements on the responses of the reviewers on the critical appraisal questions, there will be deliberations between the two reviewers. The following data were obtained from each study: Title, author 
and year of publications; total population for the study and the population for each treatment arm; and number of patients with success/failure clinical response. Reports on the secondary outcome of relative risk and serious adverse events were also extracted where available.

The analytic approach and software provided by the Cochrane Collaboration was used for all analyses. (Review Manager (RevMan) [Computer program]. Version 5.0. Copenhagen: The Nordic Cochrane Centre, The Cochrane Collaboration, 2008. Cochrane Centre, Copenhagen, Denmark). Relative risks (RRs) for studies with at least 1 occurrence in either study group for the outcome data in both groups were excluded from the meta-analysis of that outcome. Pooled RR and 95\% confidence interval (CI) for the clinical success on the treatment of impetigo, secondarily infected dermatitis, and secondarily infected traumatic lesions using both fixed-and random effects models reported. Findings were considered to be statistically significant if the test for overall effects has a $\mathrm{P}$ value of less than 0.05 . The risk estimates and confidence intervals were illustrated using forest plots. Heterogeneity was assessed through the $\mathrm{x} 2$ test with the methods of Mantel-Haenszel and quantified using the I2 test. All possible sources of material were sought to ensure lack publication bias.

A sensitivity analysis was done and the results showed no significant differences among the overall outcomes between per protocol patients, per protocol bacteriological, intention to treat patients and intention to treat bacteriological. Thus, these data were not included.

\section{Results}

\section{7-Day Efficacy Results among Per-Protocol Patients}

Considering per protocol patients at the end of the treatment (approximately 7 days), a pooled RR of 1.13 (0.97-1.31) was found in favour to retapamulin on clinical success of impetigo or other uncomplicated superficial skin infections versus other treatment used. The forest plot of 7-day efficacy among per-protocol patients with impetigo or with other uncomplicated superficial skin infections treated with retapamulin versus other treatment for the meta-analysis with heterogenous results among three studies, with an $\mathrm{I}^{2}=93 \%$.

\section{4-Day Efficacy Results among Per-Protocol Patients}

A pooled RR of 1.01 (0.94-1.09) was found in favour to retapamulin on clinical success of impetigo or other uncomplicated superficial skin infections after follow up (approximately 14 days) versus other treatment used. Figure 2 presents the forest plot for the meta-analysis with heterogenous results among five studies included, with an $\mathrm{I}^{2}=84 \%$.

\section{7-Day Efficacy Results among Per-Protocol Bacteriologic Patients}

In the forest plot of 7-day efficacy among per-protocol bacteriologic patients with impetigo or with other uncomplicated superficial skin infections treated with retapamulin versus other treatment, at the end of the treatment among patients with bacteriologic results, metaanalysis results shows heterogenous results among three studies included, with $\mathrm{I}^{2}=93 \%$. RR results of $1.16(0.96$ 1.39) favours retapamulin in clinical success treatment of impetigo or other uncomplicated superficial skin infections versus other treatment.

\section{4-Day Efficacy Results among Per-Protocol Bacteriologic Patients}

Among patients with bacteriologic results with follow up (approximately 14-days), meta-analysis results shows in figure 2 found heterogenous results among three studies included, with $\mathrm{I}^{2}=84 \%$. RR results of 1.06 (0.96-1.17) favours retapamulin in clinical success treatment of impetigo or other uncomplicated superficial skin infections versus other treatment.

\section{7-Day Efficacy Results among Intention-to-Treat Patients}

Three studies were included to measure the efficacy of retapamulin compared with other treatment in success clinical response on impetigo and other complicated superficial skin infections. A pooled RR of 1.12 (0.961.31) was instituted. The forest plot of 7-day efficacy among intention-to-treat patients with impetigo or with other uncomplicated superficial skin infections treated with retapamulin versus other treatment presents the studies were heterogenous, with an $\mathrm{I}^{2}=91 \%$.

\section{4-Day Efficacy Results among Intention-to-Treat Patients}

All of the five studies were included in the analysis for the 14-day efficacy results of retapamulin versus other treatment among intention-to-treat patients with impetigo or with other uncomplicated superficial skin infections. A pooled RR of 1.04 (0.96-1.12) was determined. Figure 3 shows the forest plot for the meta-analysis. The studies were heterogenous, with an $\mathrm{I}^{2}=81 \%$. 


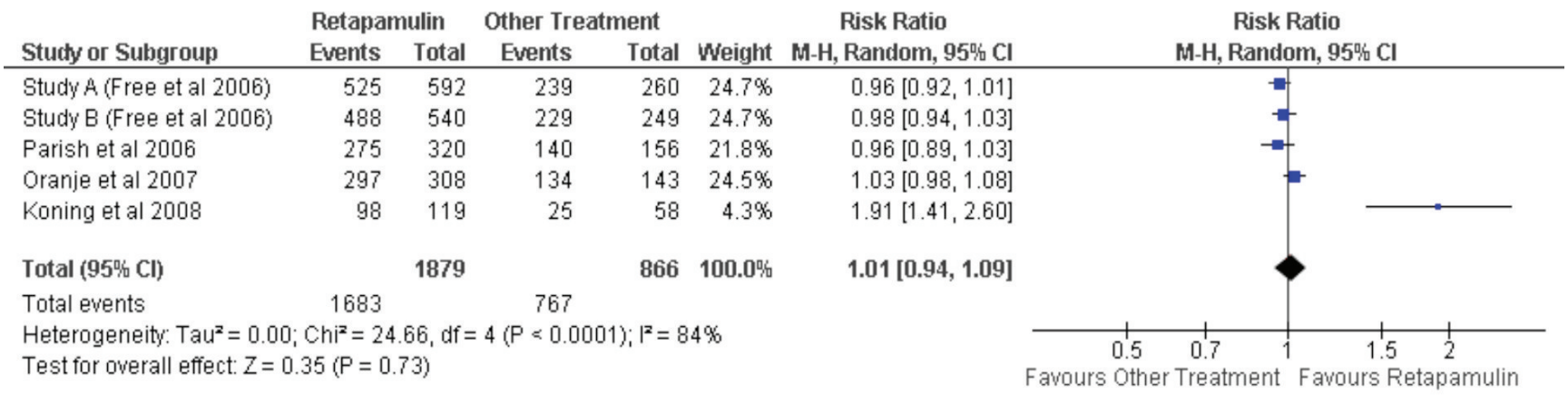

Figure 1. Forest plot of 14-day efficacy among per-protocol patients with impetigo or with other uncomplicated superficial skin infections treated with retapamulin versus other treatment.

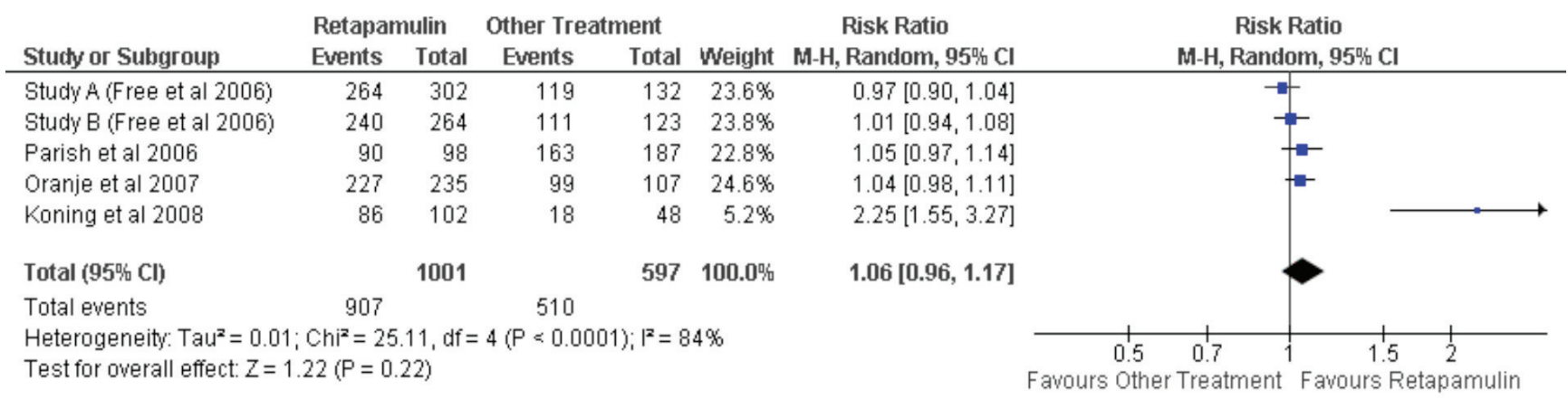

Figure 2. Forest plot of 14-day efficacy among per-protocol bacteriologic patients with impetigo or with other uncomplicated superficial skin infections treated with retapamulin versus other treatment.

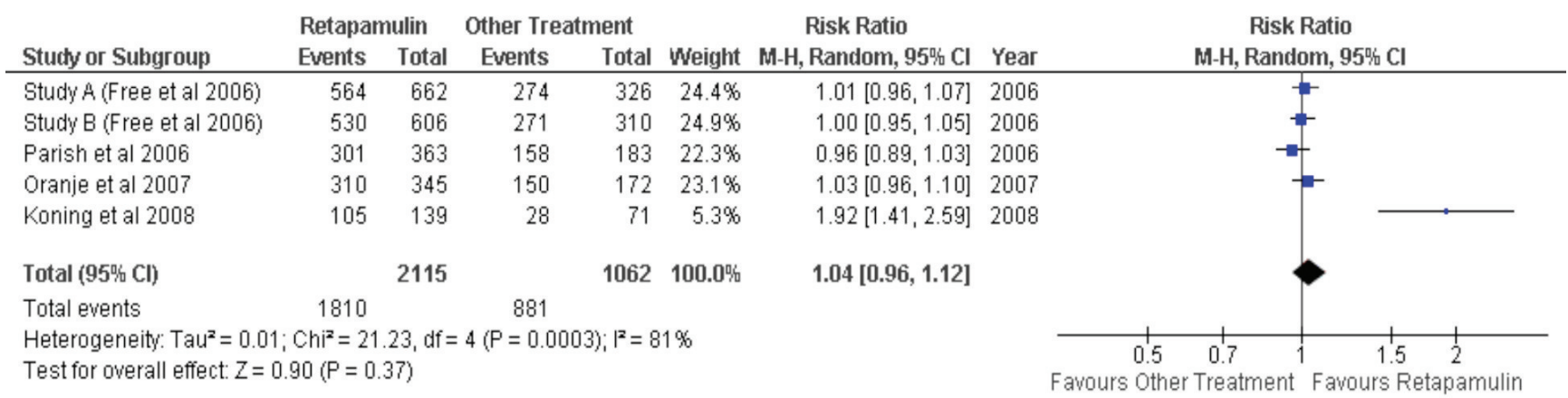

Figure 3. Forest plot of 14-day efficacy among intention-to-treat patients with impetigo or with other uncomplicated superficial skin infections treated with retapamulin versus other treatment.

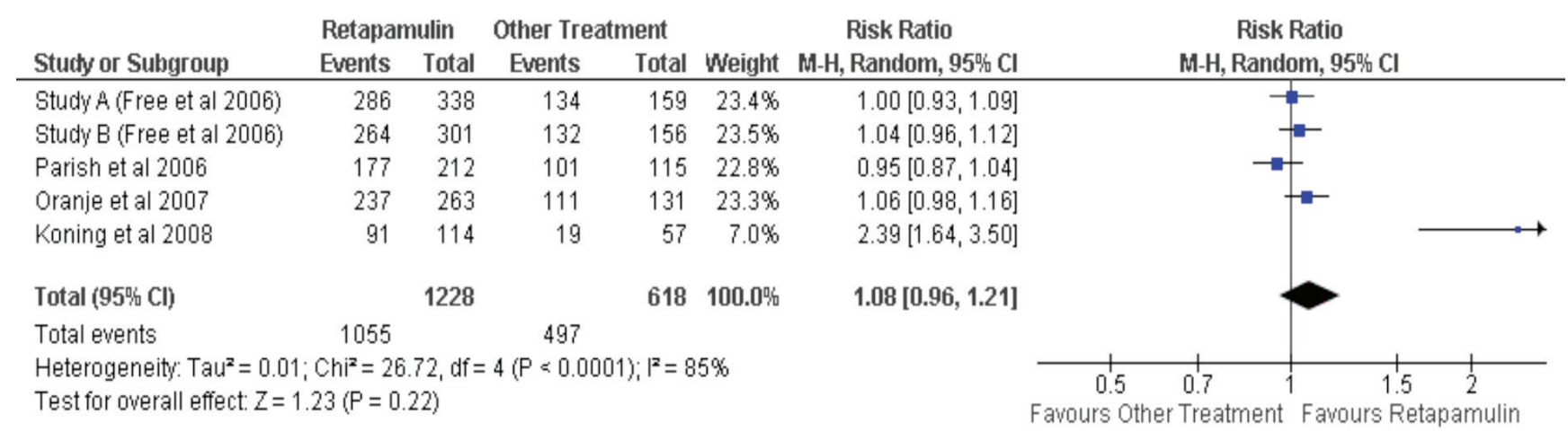

Figure 4. Forest plot of 14-day efficacy among intention-to-treat bacteriologic patients with impetigo or with other uncomplicated superficial skin infections treated with retapamulin versus other treatment. 


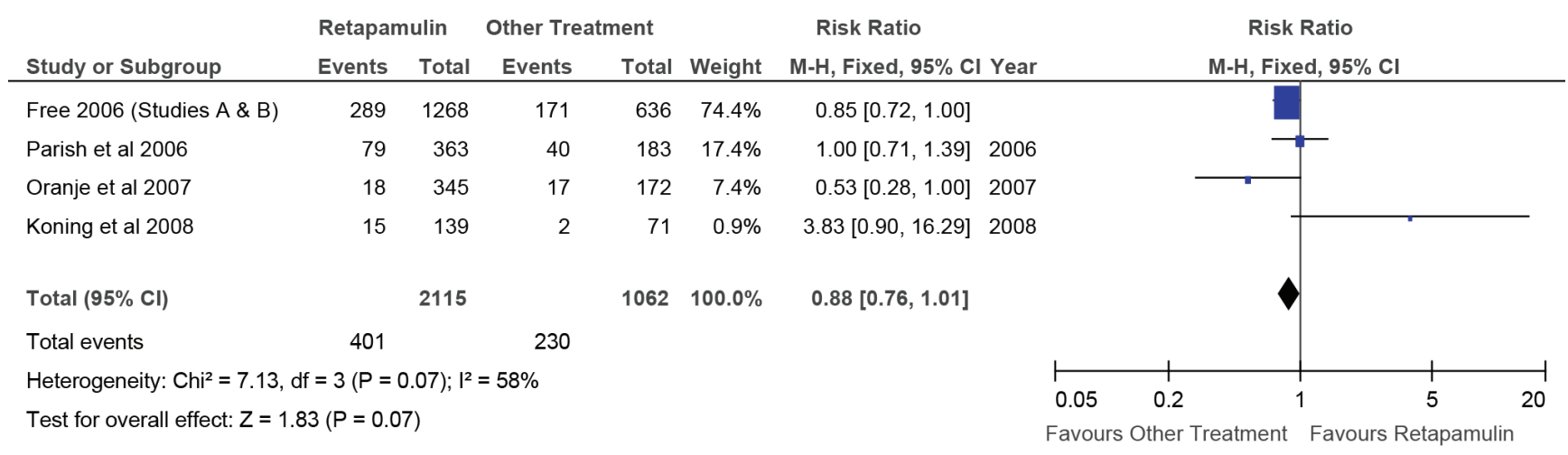

Figure 5. Forest plot of any adverse events among patients with impetigo or with other uncomplicated superficial skin infections treated with retapamulin versus other treatment.

Table 1. Most common adverse events among per-protocol patients by treatment groups

\begin{tabular}{|c|c|c|c|c|c|c|c|c|c|}
\hline \multirow[t]{2}{*}{ Adverse Events } & \multicolumn{2}{|c|}{$\begin{array}{l}\text { Retapamulin } \\
(n=2115)\end{array}$} & \multicolumn{2}{|c|}{$\begin{array}{l}\text { Cephalexin } \\
(n=819)\end{array}$} & \multicolumn{2}{|c|}{$\begin{array}{l}\text { Sodium Fusidate } \\
\qquad(\mathrm{n}=172)\end{array}$} & \multicolumn{2}{|c|}{$\begin{array}{c}\text { Placebo } \\
(n=76)\end{array}$} & \multirow[t]{2}{*}{ p-value } \\
\hline & no. & $\%$ & no. & $\%$ & no. & $\%$ & no. & $\%$ & \\
\hline Any adverse events & 458 & 21.65 & 205 & 25.03 & 25 & 14.53 & 18 & 23.68 & $0.018(\mathrm{~S})$ \\
\hline Headache & 38 & 1.8 & 16 & 1.95 & 0 & 0 & 0 & 0 & 0.216 \\
\hline Diarrhea & 31 & 1.47 & 22 & 2.69 & 2 & 1.16 & 0 & 0 & 0.115 \\
\hline Application site irritation & 30 & 1.42 & 4 & 0.49 & 0 & 0 & 1 & 1.32 & 0.063 \\
\hline Nasopharyngitis & 27 & 1.28 & 7 & 0.85 & 0 & 0 & 0 & 0 & 0.455 \\
\hline Application site pruritus & 21 & 0.99 & 3 & 0.37 & 0 & 0 & 1 & 1.32 & 0.158 \\
\hline Nausea & 19 & 0.9 & 15 & 1.83 & 0 & 0 & 0 & 0 & 0.095 \\
\hline Pruritus & 13 & 0.61 & 3 & 0.37 & 0 & 0 & 1 & 1.32 & 0.352 \\
\hline Pyrexia & 11 & 0.52 & 3 & 0.37 & 1 & 0.58 & 1 & 1.32 & 0.38 \\
\hline Abdominal pain & 9 & 0.43 & 5 & 0.61 & 0 & 0 & 1 & 1.32 & 0.367 \\
\hline Rhinitis & 6 & 0.28 & 0 & 0 & 0 & 0 & 1 & 1.32 & 0.123 \\
\hline Hyperglycaemia & 5 & 0.24 & 5 & 0.61 & 0 & 0 & 1 & 1.32 & 0.11 \\
\hline Urinary tract infection & 5 & 0.24 & 2 & 0.24 & 0 & 0 & 0 & 0 & 1 \\
\hline Urine analysis abdominal & 5 & 0.24 & 0 & 0 & 4 & 2.33 & 1 & 1.32 & $0.000(\mathrm{~S})$ \\
\hline Pain in extremity & 4 & 0.19 & 3 & 0.37 & 0 & 0 & 1 & 1.32 & 0.21 \\
\hline Application site paraesthesiae & 4 & 0.19 & 0 & 0 & 0 & 0 & 1 & 1.32 & $0.001(\mathrm{~S})$ \\
\hline Excoriation & 3 & 0.14 & 1 & 0.12 & 0 & 0 & 2 & 2.63 & $0.020(\mathrm{~S})$ \\
\hline Impetigo & 3 & 0.14 & 0 & 0 & 4 & 2.33 & 1 & 1.32 & $0.000(\mathrm{~S})$ \\
\hline
\end{tabular}

\section{7-Day Efficacy Results among Intention-to-Treat} Bacteriologic Patients

The pooled RR was $1.19(0.98-1.43)$ for the result in forest plot of 7-day efficacy of the retapamulin versus other treatment on clinical success among intention-to-treat bacteriologic patients with impetigo or other uncomplicated superficial skin infections. Three studies were found heterogenous, with $\mathrm{I}^{2}=91 \%$.

\section{4-Day Efficacy Results among Intention-to-Treat Bacteriologic Patients}

Forest plot results presented in figure 4 for the clinical success of the retapamulin versus other treatment on patients with impetigo or other superficial skin infections. Pooled RR of 1.08 (0.96-1.21) was found. Heterogeneity of the results was also shown, with $\mathrm{I}^{2}=85 \%$.

\section{Tolerability}

Retapamulin was well tolerated by patients in clinical trials and most adverse events were of mild to moderate severity. (21) Incidence of individual adverse events among perprotocol patients, regardless of causality, were low in all treatment groups, and the majority of adverse events had an incidence of $\leq 1 \%$.(21) The most common adverse events 
in the retapamulin group were headache $(1.8 \%)$, diarrhea (1.5\%), application site irritation (1.4\%) and nasopharyngitis (1.3\%). Adverse events were found highest among those patients who received cephalexin $(25.03 \%)$, followed by placebo (23.68\%) while the sodium fusidate had the lowest incidence of adverse events (14.53\%) $(\mathrm{P}=0.018)$. The common adverse events were summarized in Table 1.

Comparing any adverse events experienced by patients included in the study treated with retapamulin and to those treated with other drugs, a pool had RR was found 0.88 (0.76-1.01). The four studies included (studies A and B of Free et al combined) were homogeneous ( $\mathrm{P}=0.07)$, with slight higher $\mathrm{I}^{2}=58 \%$ (Figure 5).

\section{Discussion}

This meta-analysis included five randomized trials done in children and adults with impetigo or other uncomplicated superficial skin infections. The dosage and administration of topical retapamulin $1 \%$ ointment is indicated for use in adult and pediatric patients aged $\geq 9$ months and the regimen is to apply retapamulin onto the affected area twice daily for 5 days. The total area for treatment should be $\leq 100 \mathrm{~cm}^{2}$. The included trials assessed the clinical response rate (success or failure) at the end of the treatment duration and during follow up.

Individual effect of retapamulin on the clinical success on per-protocol patients with impetigo or other uncomplicated superficial skin infections after 7 days found no statistical significant difference when compared to cephalexin (RR 95\% CI: 0.98-1.03) but with statistically significant difference when compared to sodium fusidate (RR 95\% CI: 1.01-1.10) and placebo (RR 95\% CI: 1.322.14). Similarly, follow up after two weeks among patients with impetigo or with secondary skin infections who adhered to the protocol, efficacy of retapamulin found no statistical significance compared to cephalexin in three trials (RR 95\% CI: 0.92-1.01; RR 95\% CI: 0.94-1.03; RR 95\% CI: $0.89-1.03$ ) and among those treated with sodium fusidate (RR 95\% CI: 0.98-1.08), while with relatively higher clinical success among those treated with placebo (RR 95\% CI: 1.41-2.60). In the same manner, patients who adhered to the protocol provided bacteriologic specimen, effects of retapamulin versus the other treatment on the clinical success on impetigo or other skin infections had almost the same results.

Among intention-to-treat patients who had impetigo or with other uncomplicated superficial skin infections, cephalexin (RR 95\% CI: 0.95-1.06) and sodium fusidate (RR 95\% CI: 0.95-1.07) had no statistically significant difference on clinical success compared to retapamulin after seven days. Relatively higher clinical success was found among those treated with retapamulin than those treated with placebo (RR 95\% CI: 1.30-2.07). After one week of follow up, there are still no significant difference established on the clinical success of retapamulin compared to cephalexin in three trials (RR 95\% CI: 0.96-1.07; RR 95\% CI: $0.95-$ 1.05; RR 95\% CI: 0.89-1.03) and sodium fusidate (RR 95\% CI: 0.96-1.10). On the other hand, comparatively significant lower clinical success was noted among patients treated with placebo (RR 95\% CI: 1.41-2.59) than those patients treated with retapamulin. Surprisingly, accounting for the intention to treat bacteriologic effects of retapamulin in patients with impetigo or with other skin infections, there was moderately higher clinical success compared to those treated with sodium fusidate (RR 95\% CI: 1.01-1.16) after seven days however, no statistically significant difference was noted after 14 days of treatment (RR 95\% CI: 0.981.16). Discrepancy might be due to the large decrease in the bacteriologic specimen provided or submitted after follow up among those treated with retapamulin. Moreover, there were no significant differences on the clinical success among those treated with retapamulin and those treated with cephalexin after seven days (RR 95\% CI: 0.96-1.09) and after 14 days of follow up (RR 95\% CI: 0.93-1.09; RR 95\% CI: 0.96-1.12; RR 95\% CI: 0.87-1.04), while placebo had relatively lower effects on clinical success for both seven days (RR 95\% CI: 1.37-2.37) and follow up of 14 days (RR 95\% CI: 1.64-3.50).

Among adverse events, higher incidence were noted among those treated with cephalexin and placebo while the sodium fusidate had the lowest incidence of symptoms. Overall incidence of adverse events shows retapamulin was well tolerated and safe for those patients in clinical trials and most adverse reactions were of mild to moderate severity. Relative lower incidence of any adverse events was found among patients with impetigo or with secondary skin infections treated with retapamulin than treated with other treatment; however this was not statistically significant ( $\mathrm{P}=0.07 ; 95 \% \mathrm{CI}$ 0.76-1.01). Noteworthy to mention is that there were no serious and fatal adverse events among patients treated in the trials included.

In summary, this study found no significant difference in the clinical success after seven days and follow up among per-protocol-patients, bacteriogical confirmed patients and 
intention-to-treat patients with impetigo and other secondary infected traumatic lesions, treated with retapamulin and those patients treated with other regimen. Although, no statistically significant difference were noted among the trials, the direction of beneficial effect in the clinical success favors those patients treated with retapamulin as shown on the confidence interval for RR. Also noted on the results, the heterogeneity is high. For instance, the study by Koning et al 2008 used placebo only while Oranje et al 2007 used the topical sodium fusidate. Thus, the effect of retapamulin is superior against placebo but not with sodium fusidate. The population profile particularly the age have may also affected the heterogeneity. One study included pediatric patients while the others have adults. Sample size may also have effects. This study have limitations, such as: limited source of data with the same measurement outcome; the similarity of inclusion criteria; and high heterogeneity of data. The results of this study are just a small step to a better understanding of the applicability of retapamulin. Hence, recommendation in future studies with variety of measurement outcome should be included.

\section{Conclusion}

Retapamulin is comparable to oral cephalexin and sodium fusidate as a treatment option for impetigo and other uncomplicated superficial skin infections and the results tender to favor retapamulin over the others. Incidence of adverse events also showed relatively lower proportion in retapamulin than cephalexin nor placebo groups but higher than the sodium fusidate group. There are also no social-economic issues affecting applicability of treatment which is cost comparable when comparing to other drugs (cephalexin or sodium fusidate). Thus, the introduction of retapamulin as an available alternative option in the treatment of impetigo and other uncomplicated superficial skin infections seem to be acceptable. However, more and bigger clinical studies involving pediatric patients in varied race and age is recommended to evaluate the efficacy of retapamulin and also compare it with other drugs as medication, such as mupirocin, silver sulfadiazine and other topical antimicrobials. This will hopefully provide additional information and a more complete view on the efficacy, safety and tolerability of retapamulin whose clinical benefits on impetigo and other uncomplicated superficial skin infections is comparable.

\section{Acknowledgement}

This study was supported by Dr. Orpheus C. Monakil, FPPS and Dr. Melchor Victor G. Frias, FPPS, De La Salle University of Medical Center, Department of Pediatrics, Dasmariñas, Cavite, Philippines.

\section{References}

1. Perera G, Hay R. A guide to antibiotic resistance in bacterial skin infections. J Eur Acad Dermatol Venereol. 2005; 19: 531-45.

2. Cole C, Gazewood J. Diagnosis and treatment of impetigo. Am Fam Physician. 2007; 75: 859-64.

3. Hedrick J. Acute bacterial skin infections in pediatric medicine: current issues in presentation and treatment. Pediatr Drugs. 2003; 5 (Suppl 1): 35-46.

4. Stulberg DL, Penrod MA, Blatny RA. Common bacterial skin infections. Am Fam Physician. 2002; 66: 119-24.

5. Stevens DL, Bisno AL, Chambers HF, Everett ED, Dellinger P, Goldstein EJ, et al. Practice guidelines for the diagnosis and management of skin and soft-tissue infections. Clin Infect Dis. 2005; 41: 1373-406.

6. Koning S, Verhagen AP, van Suijlekom-Smit LW, Morris A, Butler $\mathrm{CC}$, van der Wouden JC. Interventions for impetigo. Cochrane Database Syst Rev. 2004; (2): CD003261.

7. Laube S, Farrell AM. Bacterial skin infections in the elderly: diagnosis and treatment. Drugs Aging. 2002; 19: 331-42.

8. Roberts S, Chambers S. Diagnosis and management of Staphylococcus aureus infections of the skin and soft tissue. Intern Med J. 2005; 35 (Suppl 2): S97-105.

9. Ladhani S, Garbash M. Staphylococcal skin infections in children: rational drug therapy recommendations. Pediatr Drugs. 2005; 7: 77102.

10. Electronic Medicines Compendium [homepage on the Internet]. Fucidin (fusidic acid) cream: UK prescribing information [Accessed 2007 Oct 9]. Available from: http://emc.medicines.org.uk/.

11. Electronic Medicines Compendium [homepage on the Internet] Bactroban ${ }^{\circledR}$ (mupirocin) 2\% ointment: UK prescribing information [Accessed 2007 Oct 9]. Available from: http://emc.medicines.org. $\mathrm{uk} /$.

12. GlaxoSmithKline [homepage on the Internet]. Bactroban Ointment ${ }^{\circledR}$ (mupirocin 2\% ointment): US prescribing information [Accessed 2007 Oct 9]. Available from: http://us.gsk.com/.

13. Afset JE, Maeland JA. Susceptibility of skin and soft-tissue isolates of Staphylococcus aureus and Streptococcus pyogenes to topical antibiotics: indications of clonal spread of fusidic acid-resistant Staphylococcus aureus. Scand J Infect Dis. 2003; 35: 84-9.

14. Shah M, Mohanraj M. High levels of fusidic acid-resistant Staphylococcus aureus in dermatology patients. Br J Dermatol. 2003; 148: 1018-20.

15. Dobie D, Gray J. Fusidic acid resistance in Staphylococcus aureus. Arch Dis Child. 2004; 89: 74-7.

16. Cookson BD. Mupirocin resistance in staphylococci. J Antimicrob Chemother. 1990; 25: 497-501.

17. Cookson BD. The emergence of mupirocin resistance: a challenge to infection control and antibiotic prescribing practice. J Antimicrob 
Chemother. 1998; 41: 11-8.

18. Deshpande LM, Fix AM, Pfaller MA, Jones RN; SENTRY Antimicrobial Surveillance Program Participants Group. Emerging elevated mupirocin resistance rates among staphylococcal isolates in the SENTRY Antimicrobial Surveillance Program (2000): correlations of results from disk diffusion, Etest and reference dilution methods. Diagn Microbiol Infect Dis. 2002; 42: 283-90.

19. European Medicines Agency [homepage on the Internet]. Summary of product characteristics: product information (Altargo) [Accessed 2008 Mar 26]. Available from: http://www.emea.europa.eu/.
20. GlaxoSmithKline [homepage on the Internet]. Altabax ${ }^{\mathrm{TM}}$ (retapamulin $1 \%$ ointment): US prescribing information [Accessed 2007 Aug 24]. Available from: http://us.gsk.com/.

21. European Medicines Agency [homepage on the Internet]. Tiamulin: summary report from the Committee for Veterinary Medicinal Products [Accessed 2007 Oct 31]. Available from: http://www. emea.europa.eu/.

22. Pretorius J. Skin and soft-tissue infections: the early clinical presentation of soft-tissue infections may be deceptive. CME. 2010; 28: 265-9. 